\title{
Nanoscale
}

PAPER
Check for updates

Cite this: Nanoscale, 2019, 11, 7404

\section{A novel phase function describing light scattering of layers containing colloidal nanospheres $\uparrow$}

\author{
Junxin Wang, ${ }^{a}$ Changgang $\mathrm{Xu},{ }^{\mathrm{a}, \mathrm{b}}$ Annica M. Nilsson, ${ }^{\mathrm{a}}$ Daniel L. A. Fernandes ${ }^{\mathrm{a}}$ and \\ Gunnar A. Niklasson (iD *a
}

Light scattering from small particles exhibit unique angular scattering distributions, which are strongly dependent on the radius to wavelength ratio as well as the refractive index contrast between the particles and the surrounding medium. As the concentration of the particles increases, multiple scattering becomes important. This complicates the description of the angular scattering patterns, and in many cases one has to resort to empirical phase functions. We have measured the angle dependence of light scattering from a polymer layer containing sub-micron metallic and dielectric particles. The samples exhibited strongly forward and backward peaked scattering patterns, which were fitted to a number of empirical approximative phase functions. We found that a novel two-term Reynolds-McCormick (TTRM) phase function gave the best fit to the experimental data in all cases. The feasibility of the TTRM approach was further validated by good agreement with numerical simulations of Mie single scattering phase functions at various wavelengths and sizes, ranging from the Rayleigh scattering regime to the geometrical optics regime. Hence, the widely adaptable TTRM approach is able to describe angular scattering distributions of different kinds of nanospheres and nanocomposites, both in the single scattering and multiple scattering regimes.

Received 25th February 2019 Accepted 23rd March 2019

DOI: $10.1039 / \mathrm{c} 9 \mathrm{nr} 01707 \mathrm{k}$ rsc.li/nanoscale ranging from marine scattering, ${ }^{10}$ underwater wireless optical communication systems, ${ }^{11}$ ice crystals, ${ }^{12}$ to tissues, ${ }^{13}$ human dermis, ${ }^{14}$ etc.

The scattering phase function ${ }^{15}$ characterizes the scattered intensity distribution as a function of scattering angle, and is a crucial parameter for understanding the optical characteristics of the materials in the framework of radiative transfer calculations. ${ }^{16}$ However, the scattering phase function for a plane-parallel structure containing scattering particles has not been studied sufficiently. In this work, we propose a measurement methodology to obtain the phase function of a light scattering layer in order to fit the angular dependence to empirical relations. We consider a layer containing functional light scattering nanoparticles, i.e. metallic $\mathrm{Au}$, dielectric $\mathrm{Fe}_{3} \mathrm{O}_{4}$, and $\mathrm{TiO}_{2}$ nanospheres. These nanomaterials have found extensive applications in optics, magnetism, catalysis, sensing, etc. Au is a metallic plasmonic material. In nanoparticles, the free electron gas can be excited by incident light to collective resonances, manifestied as strongly localized surface plasmon modes. ${ }^{17} \mathrm{Au}$ particles are used in applications like surface enhanced Raman spectroscopy, plasmonic sensing, photothermal and plasmon-assisted photochemical reactions. ${ }^{18}$ Magnetic $\mathrm{Fe}_{3} \mathrm{O}_{4}$ particles have been used in biosensors, exploiting optical variations under an applied magnetic field. ${ }^{19}$ $\mathrm{TiO}_{2}$ is a widely used pigment and sun-screen additive, which also has been employed as a scattering layer in dye-sensitized 
solar cells so that the path length of light is increased to facilitate a better absorption due to adsorbed dye molecules. ${ }^{15,20}$

The scattering distribution of a single isolated nanoparticle can be obtained by Mie theory using the scattering matrix ${ }^{21}$ approach. However, it is only valid for single scattering and the shape of the particles must be spherical and have a smooth surface. In reality, light scattering particles have irregular shapes with rough surfaces, for example marine scatterers and scatterers in tissue and blood. High pigment concentrations as well as particle aggregation are also common and in these cases multiple scattering effects will modify the phase function. Advanced methods like Monte Carlo modeling $^{22,23}$ and Finite-difference time-domain (FDTD) ${ }^{24}$ require complex calculations. Several empirical phase function approximations have been developed in order to assess their feasibility for describing the single and multiple scattering scenarios. One of the most widely used analytic phase functions is the one-term Henyey-Greenstein (HG) phase function, which was first used in astrophysics. ${ }^{25}$ However, a major deficiency of $\mathrm{HG}$ is that it fails to describe the glory, ${ }^{26}$ for example caused by the backscattering of sunlight from small droplets of water, which is predicted by Mie theory. ${ }^{27}$ The twoterm Henyey-Greenstein (TTHG) function, provides a better approximation for the backscattering peak that exists for many realistic particle distributions, for example marine ones. ${ }^{8}$ Cornette and Shanks, ${ }^{28}$ defined a single parameter phase function, which converges to the Rayleigh phase function for small particles and to the Henyey-Greenstein phase function for larger ones. Another alternative phase function was suggested by Reynolds and McCormick, ${ }^{29}$ and was found to fit well to data from several biological specimens. It works especially well for highly anisotropic scattering patterns. Forand and Fournier, ${ }^{30}$ proposed a phase function considering a particle size distribution of marine particulates, leading to an inverse power law behavior of scattering as a function of scattering angle.

The samples considered in the present work were considerably simpler and consisted of almost spherical nanoparticles dispersed in a layer. Our purpose was to find a facile approach to determine the effects of multiple scattering and aggregation on the phase function. By measuring the angular dependence of light scattering we could obtain a good empirical description of the multiple scattering phase function inside the layer. The experimental phase functions were compared to various empirical phase functions as well as revisions of them.

\section{Results and discussion}

\section{Angle resolved light scattering}

The synthesized $\mathrm{Au}, \mathrm{Fe}_{3} \mathrm{O}_{4}$ and $\mathrm{TiO}_{2}$ nanospheres were prepared by a wet chemistry approach and had diameters of $203 \pm$ $13 \mathrm{~nm}, 207 \pm 48 \mathrm{~nm}$, and $216 \pm 34 \mathrm{~nm}$ (see Experimental, Fig. $\mathrm{S} 1 \dagger$ ). The nanospheres were dispersed in water followed by adding 80\% PVP (compared to the weight of water). The volume fraction of the three types of nanoparticles (in water +
80 wt $\%$ PVP) was $0.066 \%$. The viscous solutions containing the particles were encapsulated between two glass slides with gaps around $80 \mu \mathrm{m}$.

We measured scattering intensity distributions from the samples by using a He-Ne laser with a wavelength of $633 \mathrm{~nm}$. A three dimensional goniometer was used to record the scattered light intensity in the forward and backward half hemispheres (Fig. S2, $\uparrow$ Experimental). The scattering intensity is presented as angle resolved scattering (ARS), ${ }^{3}$ which is defined as

$$
\operatorname{ARS}(\theta)=\frac{I(\theta)}{P_{\mathrm{i}} \times \Omega}
$$

where $P_{\mathrm{i}}$ is the incident light intensity, $\Omega$ is the solid angle of the detector and $I(\theta)$ is the scattering intensity as a function of polar angle $\theta$. Forward scattering (FS) represents the light intensity in the hemisphere in the direction that the light propagates, i.e. for polar angles in the $\left[0^{\circ}, 90^{\circ}\right]$ range, while backward scattering (BS) denotes the light intensity in the opposite hemisphere when the $\theta$ range is $\left[90^{\circ}, 180^{\circ}\right]$. The measured forward and backward scattering intensity in a half hemisphere for the three nanoparticle samples can be plotted as a function of spatial detector position (Fig. S2b, c $\dagger$ ). By mirroring the intensity to the other half hemisphere, an extended scattering distribution can be plotted, as seen in Fig. 1a-c for the forward and in Fig. S3a-c $\dagger$ for the backward hemisphere. It is clearly observed that strong peaks appear in the direct forward and backward directions, and there is a large drop in intensity towards higher angles. There is an order of magnitude higher intensity in the forward peak compared the backward peak for all three nanoparticle composites.

We then plotted measured scattering data as a function of polar angle $(\theta)$ and azimuth angle $(\varphi)$ (see Experimental). Fig. 1d-f shows ARS of forward scattering at various polar angles as a function of azimuth angle in logarithmic scale. Fig. 1d-f shows that scattering is mainly dependent on the polar angle and rather insensitive to the azimuth angle. The speckle-like pattern in Fig. 1a-c is probably caused by an inhomogeneous particle distribution. Fig. S3† shows corresponding backward scattering data as a function of polar/ azimuth angle. Since the backward scattering is lower than the forward one, higher measurement fluctuations may occur. The data show clear evidence of negligible dependence on azimuth angle, indicating that scattering is mainly dependent on polar angle. Fig. 1g-i gives the ARS of forward scattering as a function of the polar angle.

Our in-house 3D goniometer works at single wavelength at $633 \mathrm{~nm}$, and in order to characterize spectrally dependent properties of the samples, we use an angle resolved spectrometer to record the dependence on polar angle of the scattering profile at various wavelengths from $350 \mathrm{~nm}$ to $1000 \mathrm{~nm}$. Fig. $2 \mathrm{a}$ and $\mathrm{b}$ show the forward and backward scattering pattern of a $\mathrm{Fe}_{3} \mathrm{O}_{4}$-PVP layer at a wavelength of $500 \mathrm{~nm}$. The zoomed-in ARS in the insets of Fig. 2a and b show that collimated light is mainly detected at angles lower than $2^{\circ}$ (shaded 

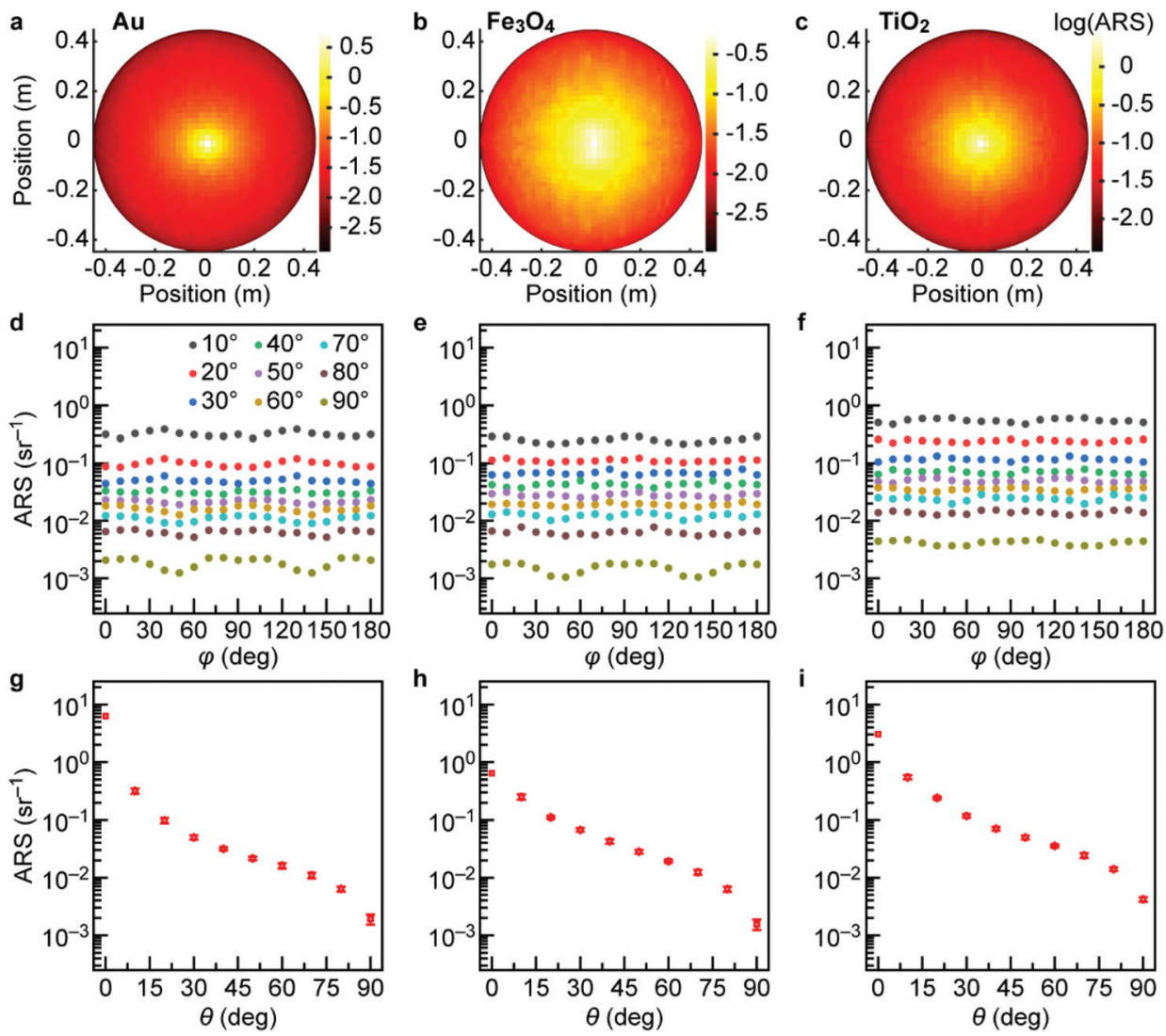

Fig. 1 (a-c) Top view of the forward angle resolved scattering (ARS) against position for three nanoparticle scattering layers (in logarithmic scale). (d-f) Forward ARS as a function of azimuth angle taken at different polar angles from $0^{\circ}$ to $90^{\circ}$ at an interval of $10^{\circ}$. (g-i) Forward ARS plotted as a function of the polar angle. The wavelength of the incident light was $633 \mathrm{~nm}$.

area). Within this interval, most of the signal comes from incident light which is not absorbed or scattered by the particles, but a minor portion results from light scattered by the nanoparticles at low angles. The latter one (the diffuse part) can be estimated by extrapolation from the intensity at angles larger than $2^{\circ}$ (inset in Fig. 2a and b, open circles), and is 2 to 3 orders of magnitude lower than the collimated incident light. The full angle resolved diffuse scattering of $\mathrm{Fe}_{3} \mathrm{O}_{4}$ is shown in Fig. 2c. The corresponding plots for $\mathrm{Fe}_{3} \mathrm{O}_{4}, \mathrm{Au}$ and $\mathrm{TiO}_{2}$ at three different wavelengths are shown in Fig. S4. $\dagger$ In order to obtain the light intensity inside the scattering layer, outer and inner angles and intensities should be correlated by Snell's law and Fresnel's equations. Firstly, we use Snell's law to obtain the angle of propagation of light inside the layer $\left(\theta_{\mathrm{i}}\right)$ from the outer scattering angle $\left(\theta_{0}\right)$ by taking account of the refractive index difference of the host (glass/(water + PVP)/ glass), $n_{\mathrm{i}}$, to that of the air, $n_{\mathrm{o}}$.

$$
\theta_{\mathrm{i}}=\arcsin \left(\frac{\sin \left(\theta_{\mathrm{o}}\right)}{\frac{n_{\mathrm{i}}}{n_{\mathrm{o}}}}\right)
$$

Then the measured ARS is used to obtain the bidirectional scattering distribution function (BSDF, denoted $f$ ) ${ }^{3}$ The difference between ARS and BSDF is the cosine correction factor, $\cos (\theta)$, to correct for the surface area at the viewing angle. The phase function describes the angular distribution of the scattered radiation inside the material and is analogous to BSDF. ${ }^{1,31}$ We also correct for the Fresnel reflectance from glass to air and obtain,

$$
f\left(\theta_{\mathrm{i}}\right)=\frac{\operatorname{ARS}\left(\theta_{\mathrm{i}}\right)}{\left[1-r\left(\theta_{\mathrm{i}}\right)\right] \cos \left(\theta_{\mathrm{i}}\right)}
$$

where $r\left(\theta_{\mathrm{i}}\right)$ denotes the Fresnel reflectance of light incident from the nanocomposite layer onto the interface with air (see Experimental).

The obtained BSDF of a $\mathrm{Fe}_{3} \mathrm{O}_{4}$ layer is shown in Fig. 2d, combining information from measurements of forward and backward scattering. It is seen that angular information of the BSDF is missing due to total internal reflection at angles larger than the critical angle. In order to bridge this gap in angles, fitting to a proper model of the phase function is required. However, it is evident that the BSDF must be very low at angles between $40^{\circ}$ and $140^{\circ}$ and values in this interval will not affect total scattering much. We choose to fit the BSDF to empirical phase functions in the range is from $1.3^{\circ}-39^{\circ}$ for the forward region and $141^{\circ}-178.7^{\circ}$ for the backward region (thick lines in Fig. 2d), thus avoiding the region where collimated transmittance and reflectance dominate. In addition, the drop in inten- 

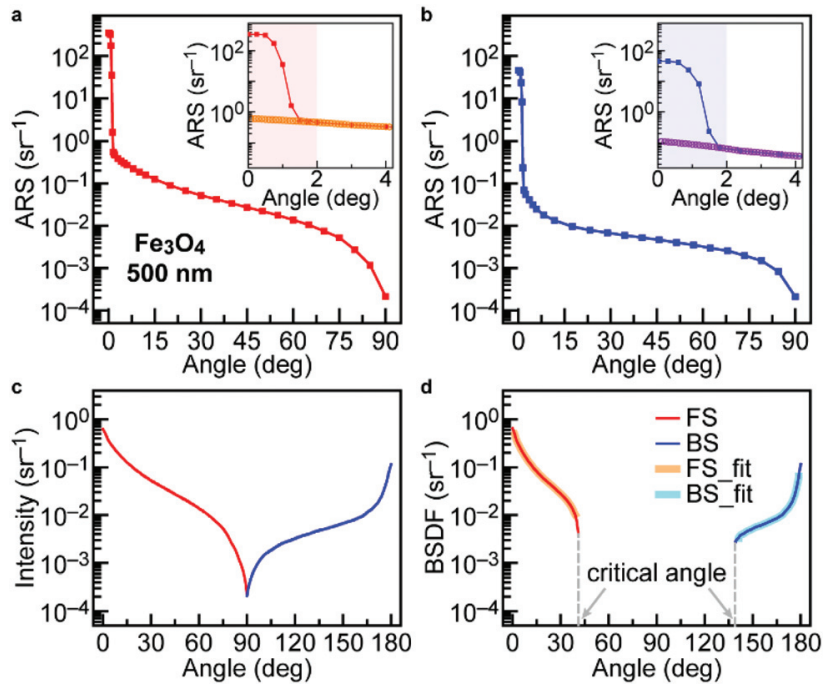

Fig. 2 (a) Forward and (b) backward ARS intensity measured by an angle resolved spectrometer on a $\mathrm{Fe}_{3} \mathrm{O}_{4}$ nanosphere sample at a wavelength of $500 \mathrm{~nm}$ as a function of scattering angle from the normal/ specular direction. The inset shows the ARS at small scattering angles. (c) Forward and backward intensity of diffuse scattering measured on the $\mathrm{Fe}_{3} \mathrm{O}_{4}$ sample at $500 \mathrm{~nm}$ as a function of polar angle. (d) Rescaled $\mathrm{BSDF}$, converting the measured intensity from (c) into the scattering distribution inside the sample, by eqn (2) and (3). The broad orange/cyan curves denote the angular ranges used for fitting.

sity close to the critical angles may be due to the detector sensitivity (see Experimental).

\section{Comparison to empirical phase functions}

We first review a number of empirical phase functions that have been proposed previously and then use them to fit our experimental data. Reynold and McCormick (RM) proposed a phase function approximation which described highly anisotropic angular scattering distributions and has the following analytic form: ${ }^{29}$

$$
P_{\mathrm{RM}}(\theta, g, \alpha)=\frac{\alpha g\left(1-g^{2}\right)^{2 \alpha}}{\pi\left[1+g^{2}-2 g \cos (\theta)\right]^{\alpha+1}\left[(1+g)^{2 \alpha}-(1-g)^{2 \alpha}\right]}
$$

When the fitting parameter $\alpha$ equals 0.5 , it reduces to the so-called Henyey-Greenstein (HG) phase function: ${ }^{25}$

$$
P_{\mathrm{HG}}(\theta, g)=\frac{1}{4 \pi} \frac{1-g^{2}}{\left[1+g^{2}-2 g \cos (\theta)\right]^{\frac{3}{2}}}
$$

where $g$ is the asymmetry factor, ranging from backscattering $(-1)$ through isotropic scattering (0) to forward scattering (1). The HG function can also be written as an expansion of Legendre polynomials, $P_{\mathrm{n}}{ }^{28}$

$$
P_{\mathrm{HG}}(\cos \theta, g)=\sum_{n=0}^{\infty}(2 n+1) g^{n} P_{n}(\cos \theta)
$$

The advantage of the RM phase function as compared to the HG phase function is that it better reproduces strongly an- isotropic scattering angular distributions. However, both of them can fit only forward peaked or backward peaked scattering distributions, and fail for distributions that are both forward and backward peaked. In order to overcome this deficiency and better represent the backscattering peak, a twoterm modified HG phase function has been proposed (TTHG), ${ }^{10}$

$$
P_{\mathrm{TTHG}}\left(\theta, \gamma, g_{1}, g_{2}\right)=\gamma P_{\mathrm{HG}}\left(\theta, g_{1}\right)+(1-\gamma) P_{\mathrm{HG}}\left(\theta, g_{2}\right)
$$

This function has two parts with two different asymmetry factors, where $g_{1}$ is positive and $g_{2}$ is negative, in order to treat the forward and backward peaks in the phase function. The parameter $\gamma$ gives the forward scattering portion while $(1-\gamma)$ is the backward scattering portion. Guided by the TTHG, we have revised the RM function in a similar way, and we denote this function as the two-term Reynolds-McCormick or TTRM phase function:

$P_{\mathrm{TTRM}}\left(\theta, g_{1}, g_{2}, \alpha_{1}, \alpha_{2}, \gamma\right)=\gamma P_{\mathrm{RM}}\left(\theta, g_{1}, \alpha_{1}\right)+(1-\gamma) P_{\mathrm{RM}}\left(\theta, g_{2}, \alpha_{2}\right)$

Compared to TTHG, TTRM has two more fitting parameters, namely $\alpha_{1}$ and $\alpha_{2}$. Besides TTHG and TTRM, we also examined the Cornette Shanks (CS) phase function: ${ }^{28}$

$$
P_{\mathrm{CS}}(\theta, g)=\frac{3\left[1+\cos (\theta)^{2}\right]}{2\left(2+g^{2}\right)} \frac{1-g^{2}}{\left[1+g^{2}-2 g \cos (\theta)\right]^{\frac{3}{2}}}
$$

The CS phase function, has only one variable, that is the asymmetry factor $g$. When $g$ is close to 0 it converges to the Rayleigh phase function and when $g$ is close to 1 it approaches the HG phase function, therefore, it provides a realistic description of the scattering by small particles. The deficiency of the CS phase function is that it fails to reproduce the sharp forward/backward scattering peak. The Forand-Fournier phase function (FF) was first derived for scatterers in oceanic water, and considers a power law for the particle size distribution and mean index of refraction of the scattering particles: ${ }^{30}$

$$
\begin{aligned}
& P_{\mathrm{FF}}(u, v, \delta)= \\
& \quad \frac{1}{4 \pi} \frac{1}{(1-\delta)^{2} \delta^{v}}\left\{\left[v(1-\delta)-\left(1-\delta^{\nu}\right)\right]+\frac{4}{u^{2}}\left[\delta\left(1-\delta^{\nu}\right)-v(1-\delta)\right]\right\}
\end{aligned}
$$

where $v=(3-\mu) / 2, \delta=u^{2} /\left[3(n-1)^{2}\right]$, and $u=2 \sin (\theta / 2)$. Similar to the cases of TTHG and TTRM, we extend it to a two-term form (TTFF) for fitting the forward and backward peaked case. The above-mentioned phase functions emerge from physical arguments, but we also propose a mathematical analytic form for fitting forward and backward peaked scattering distributions based on a parabolic curve. This approximation is denoted as PARA:

$$
P_{\text {PARA }}(\theta, a, b, c)=\exp \left[a+b(\theta-c)^{2}\right]
$$

Omitting the less suitable one-term functions, we now have 5 different analytic approximations to the phase function, namely TTRM, TTHG, TTFF, CS and PARA, which were fitted 
to the experimental data. The quality of fit was described by the mean square logarithmic error (MSLE) criterion, which was used to find the difference of the fitted curve to the BSDF from the experiment:

$$
\text { MSLE }=\sqrt{\frac{\sum_{0}^{\theta}\left[\log \left[I\left(\theta_{\mathrm{i}}\right)\right]-\log \left[F\left(\theta_{\mathrm{i}}\right)\right]\right]^{2}}{N}}
$$

The reason to take logarithmic error is because there are orders of magnitude differences within the same scattering profiles, otherwise the fitting will be dominated by the largest values. The fitting curves (green) of the TTRM, TTHG, PARA, TTFF and CS approximations for a $\mathrm{Fe}_{3} \mathrm{O}_{4}$ sample are plotted in Fig. $3 \mathrm{a}-\mathrm{e}$, and in addition Fig. $\mathrm{S} 5 \dagger$ gives analogous data for $\mathrm{Au}$ and $\mathrm{TiO}_{2}$ samples, all at $500 \mathrm{~nm}$. Among them, TTRM exhibits the lowest MSLE for all the samples at all wavelengths, followed by the TTHG and PARA approximations (Fig. 3f-h). CS shows the worst fit among these five methods, probably because it only contains one fitting parameter, $g$, and is more suitable for fitting Rayleigh like scattering phase functions. The one-term functions HG, RM and FF fail to reproduce the backscattering peaks (Fig. S6†).

It is seen that TTRM and TTHG phase functions have the lowest MSLE among these approximations. The largest discrepancies frequently occur close to the critical angles where the experimental data in several cases exhibit a sharp drop. However, at these points experimental uncertainties may be significant and we cannot be certain that these features are physical. In addition, the TTHG function has problems to fit data close to the forward and backward directions. The comparison of TTRM and TTHG function to the experimental BSDF of $\mathrm{Fe}_{3} \mathrm{O}_{4}$ from 350 to $1000 \mathrm{~nm}$ is shown in Fig. 4. In addition, Fig. $\mathrm{S} 7$ and $\mathrm{S} 8 \uparrow$ show analogous data for the Au and $\mathrm{TiO}_{2}$ samples.

Compared to TTHG, TTRM better follows the trend of both forward and backward scattering branches, especially close to $0^{\circ}$ and $180^{\circ}$, which can be to a large extent attributed to the introduction of scattering anisotropy in both directions by the fitting parameters $\alpha_{1}$ and $\alpha_{2}$.

We now examine the fitting parameters obtained from these samples. Turning to TTRM, the forward and backward asymmetry factors $g_{1}$ and $g_{2}$, together with the two new free parameters $\alpha_{1}$ and $\alpha_{2}$ are plotted in Fig. 5a-c. The asymmetry factors are close to 1 and -1 , respectively, signifying a strongly forward and backward peaked scattering pattern. The fitted forward portion, $\gamma$, is significantly above 0.5 , indicating that forward scattering is dominant (Fig. 5d-f). The asymmetry factors and forward scattering fraction, obtained from fits to the TTHG functions, show qualitatively similar behaviours (Fig. S9†). However, we found that a higher averaged asymmetry factor leads to a lower backscatter fraction in the TTHG fits (Fig. S9d, h, $1 \dagger$ ). Because of the introduction of the parameters $\alpha_{1}$ and $\alpha_{2}$ in the TTRM function, the asymmetry factor is not the only factor determining the backscattering and hence there is no systematic relation between the backscatter fraction and the average asymmetry factor in this case (Fig. S10†).

\section{TTRM fit to Rayleigh and Mie phase functions}

It was shown above that the TTRM phase function can fit our experimental scattering distributions with good accuracy. The experimental data is influenced by multiple scattering, most probably due to aggregation of particles in the samples (Fig. S11†). In order to verify the feasibility of the TTRM function in a broader context, we now compare TTRM with the Mie
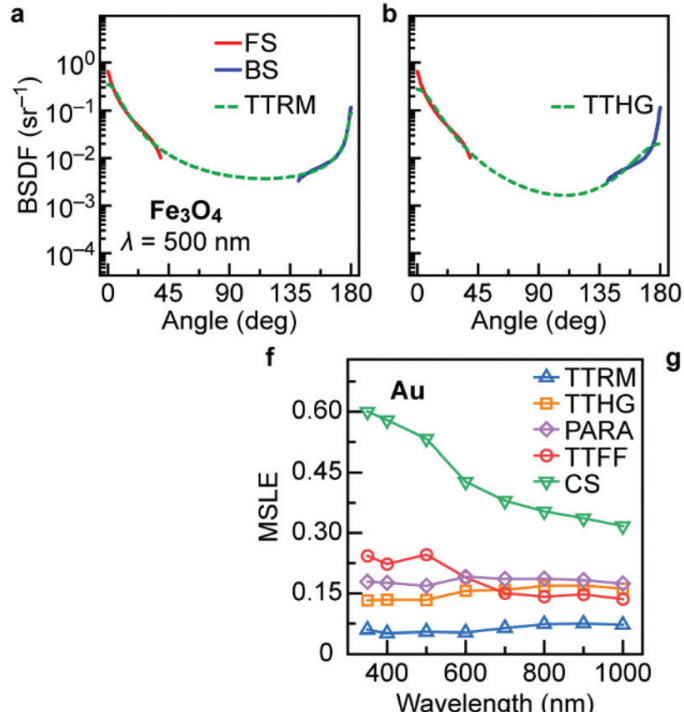
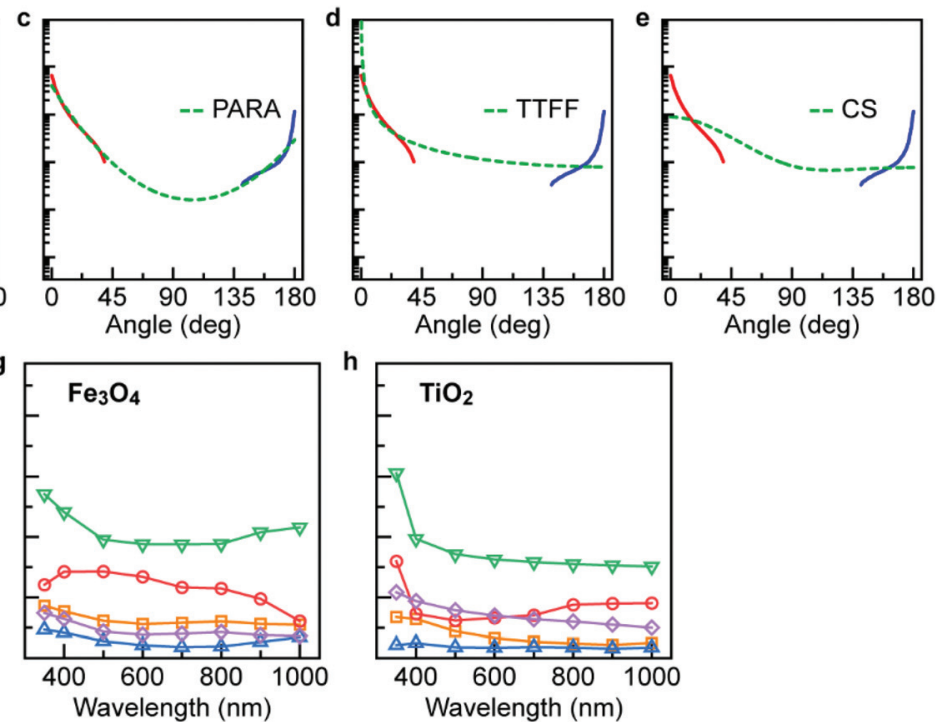

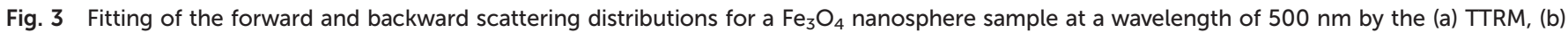

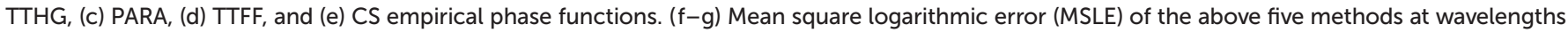
from $350 \mathrm{~nm}$ to $1000 \mathrm{~nm}$, for $\mathrm{Au}$ (f), $\mathrm{Fe}_{3} \mathrm{O}_{4}$ (g) and $\mathrm{TiO}_{2}$ (h) samples. 

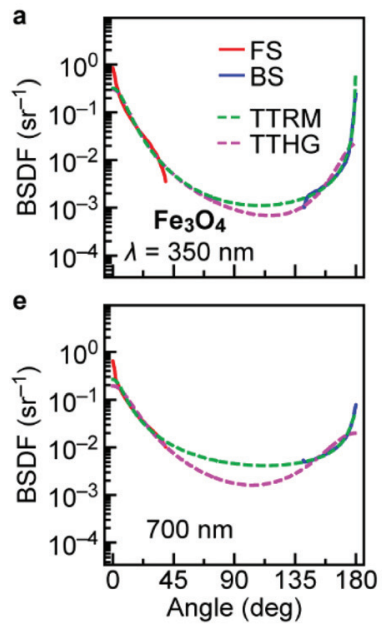
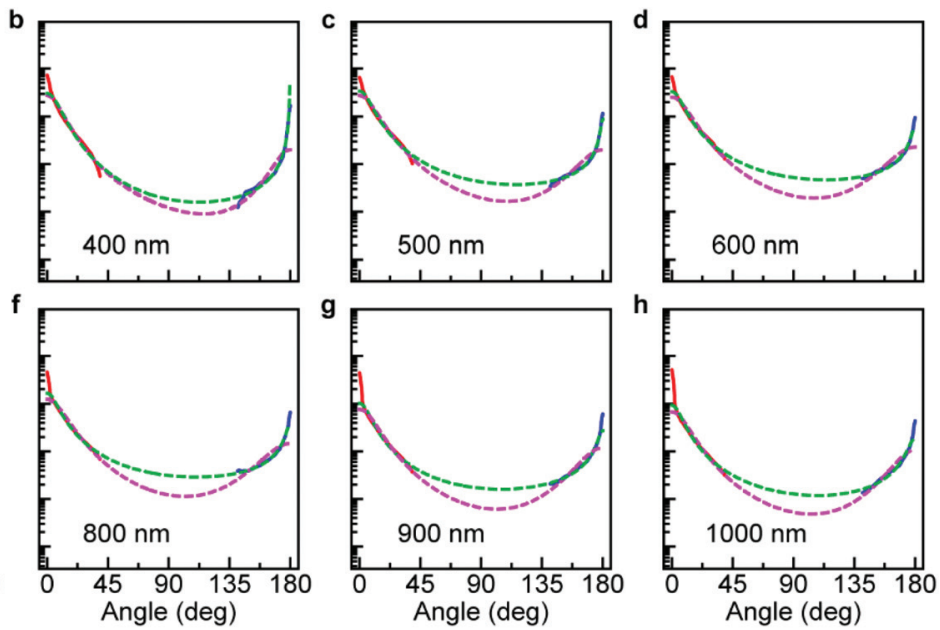

Fig. 4 Fitting of the forward and backward scattering distributions for a $\mathrm{Fe}_{3} \mathrm{O}_{4}$ sample at wavelengths from $350 \mathrm{~nm}$ to $1000 \mathrm{~nm}$ (a-h) by use of the TTHG and TTRM phase function approximations.
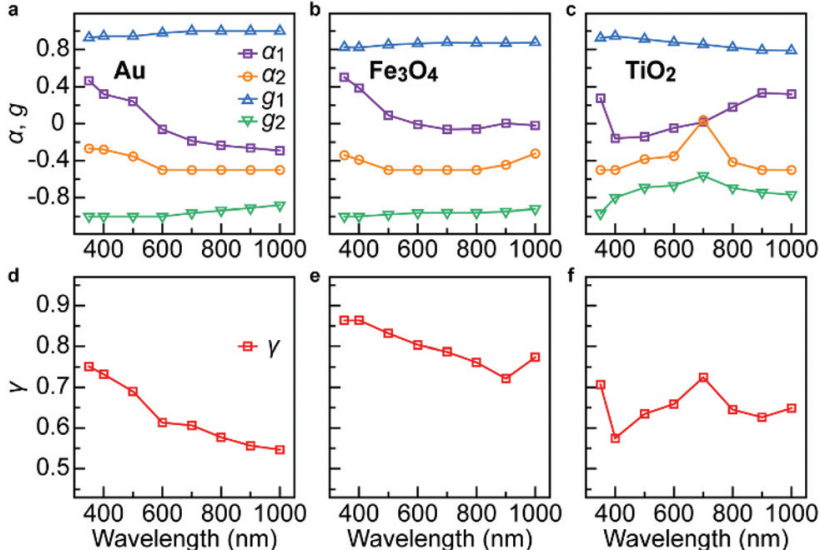

Fig. 5 Parameters obtained from fits to the TTRM function for $\mathrm{Au}$, $\mathrm{Fe}_{3} \mathrm{O}_{4}$ and $\mathrm{TiO}_{2}$ nanoparticle samples as a function of wavelength: $\alpha_{1}, \alpha_{2}$, $g_{1}, g_{2}$ for (a) $\mathrm{Au}$, (b) $\mathrm{Fe}_{3} \mathrm{O}_{4}$ and (c) $\mathrm{TiO}_{2}$ samples and the forward portion

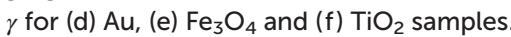

scattering phase function, that describes scattering from a single spherical particle or the single scattering regime of a nanoparticle composite. Mie theory ${ }^{15}$ was used to calculate the far-field scattering pattern around the particle. The Mie phase function considering the measured size distributions of the three types of particles, as obtained from SEM images (Fig. S1 $\dagger$ ), with average diameters about $200 \mathrm{~nm}$ are plotted and fitted by the TTRM expression as a function of wavelength from $400 \mathrm{~nm}$ to $1200 \mathrm{~nm}$ (Fig. 6). Results show that the TTRM function overlaps with the Mie phase functions in many cases, but slight discrepancies appear when there are oscillations in the Mie calculations owing to excitations of higher order eigenmodes, or normal modes. ${ }^{21}$ From the scattering phase function, one can notice that at shorter wavelengths, forward scattering is dominant while the backward portion increases with increasing wavelength.
We now explore phase functions from Mie theory for particles with diameters different from $200 \mathrm{~nm}$. The size parameter, $x=2 \pi a / \lambda$, where $a$ is the radius of the nanosphere and $\lambda$ is the wavelength of incident light, is frequently used in the field of optical scattering to define different scattering domains. For $x$ significantly less than one Rayleigh scattering predominates. For $x>20$ one enters the geometric optics regime and the intermediate regime is often called the Mie scattering regime. The Rayleigh phase function describes the angular scattering distribution of unpolarized light by small particles: ${ }^{21}$

$$
P_{\text {Rayleigh }}(\theta) \sim\left(1+\cos ^{2}(\theta)\right)
$$

The Rayleigh scattering function is symmetric, with maxima at $0^{\circ}$ and $180^{\circ}$ while minima appear at $90^{\circ}$ and $270^{\circ}$. In the Mie region, the scattering pattern becomes more anisotropic and forward scattering dominates more as the particle size increases. We present Mie calculations for a wavelength of $600 \mathrm{~nm}$, with diameters of single particles equal to 50, 150, 300,500 and $1000 \mathrm{~nm}$, corresponding to size parameters $x$ equal to $0.26,0.79,1.57,2.62$ and 5.24 and fitted them by the TTRM function (Fig. 7). Multiple peaks appear when $x$ is larger than one. The fitting in the Rayleigh region (first column), and Mie region when $x$ is smaller than unity (second column), between the TTRM and Rayleigh/Mie phase functions is excellent. The TTRM function still follows the trend when $x$ is larger than 1, although it does not describe the multiple peaks occurring due to higher order eigenmodes. However, in practical light scattering measurements, these peaks are usually smoothed due to size and shape distributions of the particles; in addition multiple scattering and the surface roughness of the particles may also contribute to this effect. Results for particles with diameters of $2000 \mathrm{~nm}(x=10.5), 4000 \mathrm{~nm}(x=21.0)$, $10000 \mathrm{~nm}(x=52.4)$ are plotted in Fig. S12, $\dagger$ illustrating the fitting capability of the TTRM also in the geometric optics regime. 

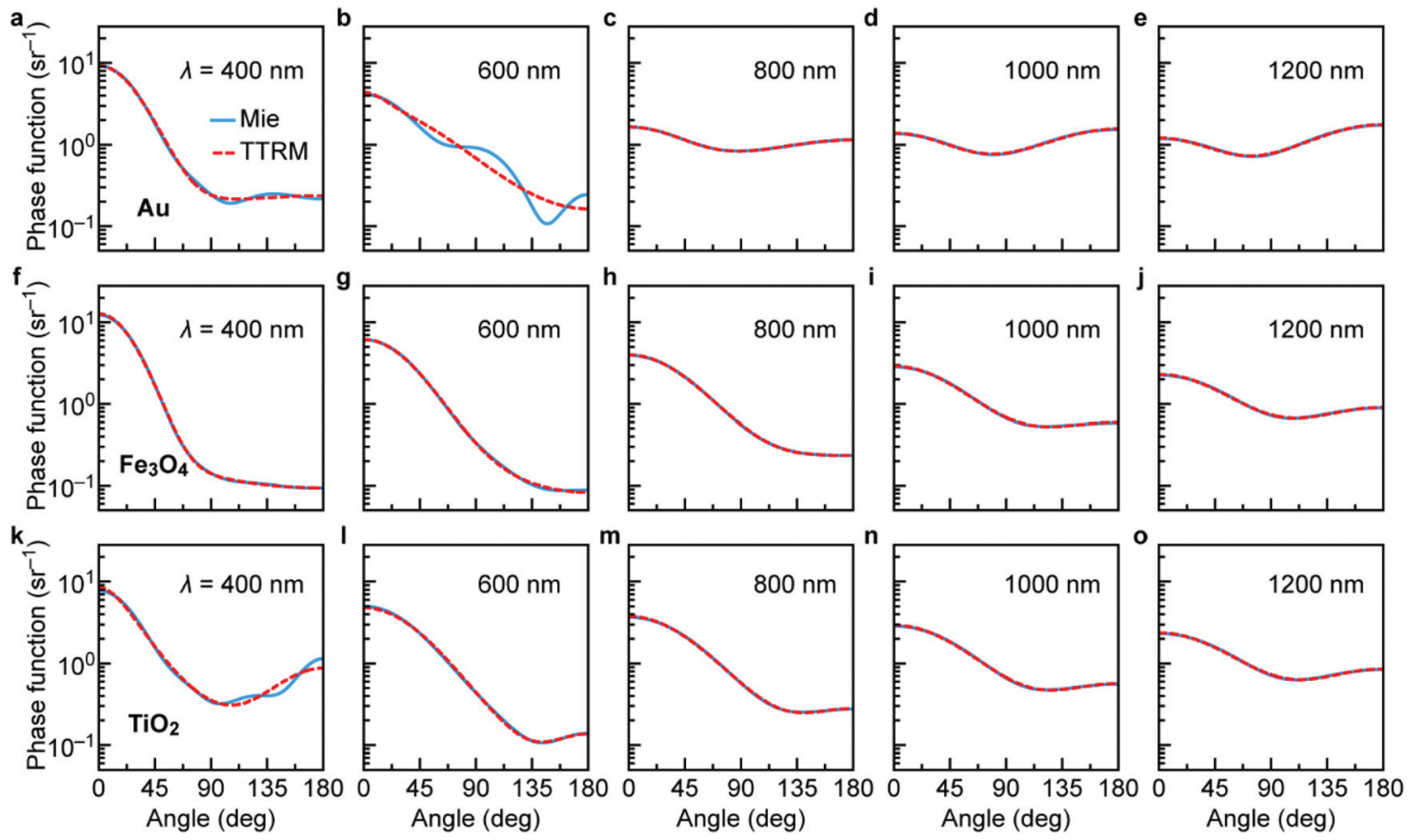

Fig. 6 Comparison of TTRM (red) phase functions with Mie (blue) phase functions for $\mathrm{Au}(\mathrm{a}-\mathrm{e}), \mathrm{Fe}_{3} \mathrm{O}_{4}\left(\mathrm{f}-\mathrm{j}\right.$ ) and $\mathrm{TiO}_{2}(\mathrm{k}-\mathrm{o})$ particles at wavelengths of $400 \mathrm{~nm}, 600 \mathrm{~nm}, 800 \mathrm{~nm}, 1000 \mathrm{~nm}$ and $1200 \mathrm{~nm}$. The Mie calculations were carried out using the experimental size distributions of nanoparticles with average diameters around $200 \mathrm{~nm}$.
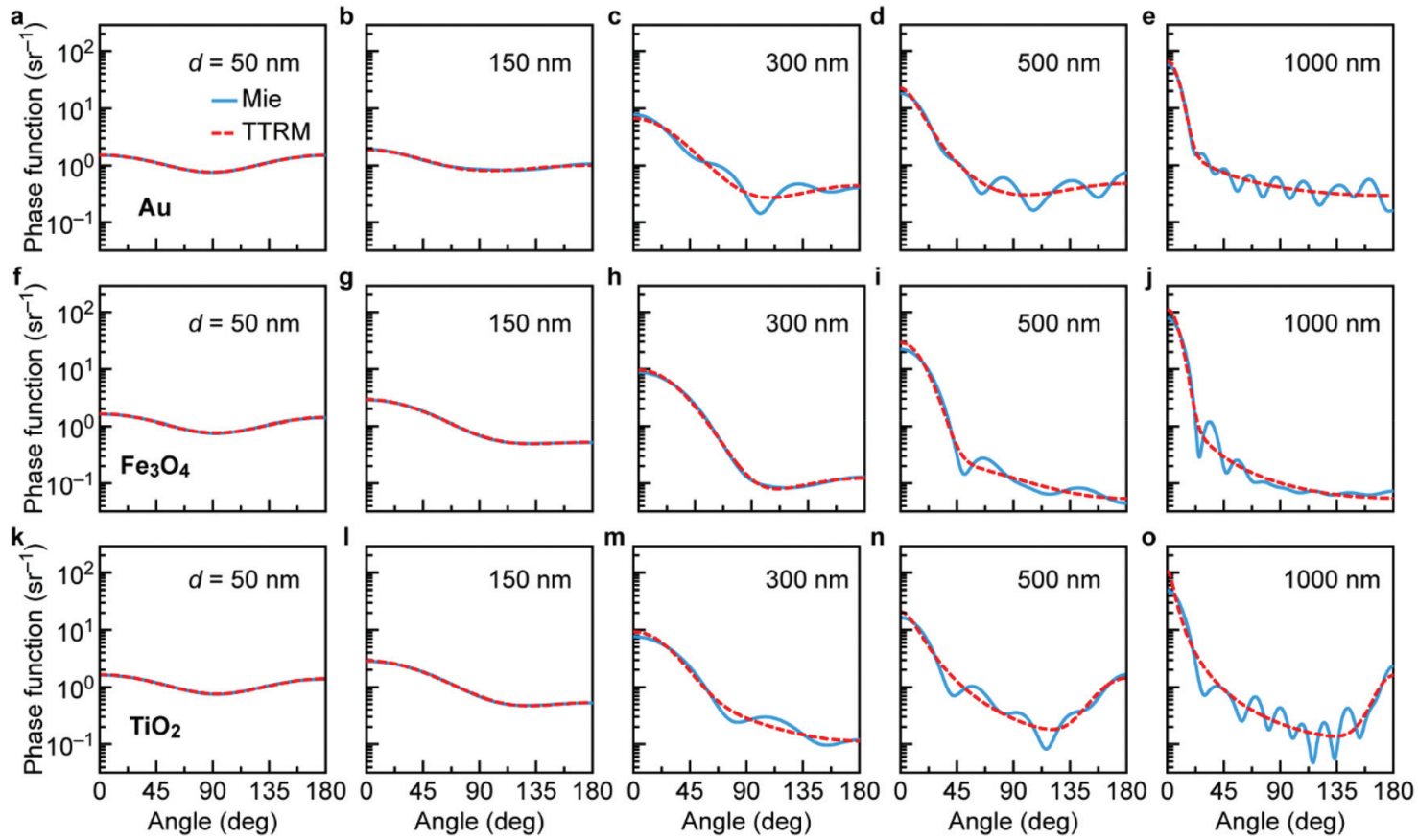

Fig. 7 Comparison of the TTRM (red) phase function with the Mie scattering model (blue) for $\mathrm{Au}(\mathrm{a}-\mathrm{e}), \mathrm{Fe}_{3} \mathrm{O}_{4}(\mathrm{f}-\mathrm{j})$ and $\mathrm{TiO}_{2}(\mathrm{k}-\mathrm{o}) \mathrm{particles}$ with diameters of $50 \mathrm{~nm}, 150 \mathrm{~nm}, 300 \mathrm{~nm}, 500 \mathrm{~nm}$ and $1000 \mathrm{~nm}$. The simulation was conducted at a wavelength of $600 \mathrm{~nm}$.

\section{Discussion}

We now discuss the physics associated with light scattering in the nanoparticle composites studied in this paper. We have found that the two-term Reynold-McCormick phase function can fit single scattering Mie calculations within a wide range of particle sizes. In the multiple scattering region a larger variety of scattering patterns can appear and the physical 
mechanisms are more complex. ${ }^{32}$ Previous approaches, like the HG and TTHG functions have particular problems with fitting strongly anisotropic scattering patterns in both forward and backward directions, such as those found experimentally here. It now remains to elucidate the physical reasons for these scattering patterns. Our samples had a low volume fraction and contained particles with diameters around $200 \mathrm{~nm}$. The single scattering from such particles was shown to be strongly directed forward in most cases (Fig. 6), and this could explain the forward scattering peaks observed in Fig. 3 and 4. Furthermore, it is observed that the backscattering peak is about an order of magnitude lower that the forward one. An important contribution to the backscattering undoubtedly comes from unscattered or low-angle scattered light, that is reflected at the back interface of the sample and subsequently forward scattered by the particles on its return path to the front surface. Multiple scattering would make the scattering pattern more isotropic ${ }^{33}$ and hence the forward and backward scattering peaks would diminish. These effects are evidently not very strong in our case. However, dependent scattering from particle clusters due to particle aggregation in our samples (Fig. S11†) cannot be ruled out. Coherent backscattering from particle clusters would enhance the backscattering peak. $^{34,35}$ In addition, aggregated particles can approximately be represented by equal volume spheres with larger sizes and this effect would also enhance the forward scattering peak.

\section{Conclusions}

In this paper, we measured the angular resolved light scattering distribution of plasmonic and dielectric nanoparticle composites as a function of wavelength from $350 \mathrm{~nm}$ to $1000 \mathrm{~nm}$. From the experimental data we could obtain an effective scattering phase function inside the composite layer. This function exhibits peaks in the forward and backward directions and the experimental data are restricted to angles closer to the surface normal than the critical angles of total internal reflection. The experimental results were compared to eight different empirical phase functions and we found that a twoterm Reynolds-McCormick (TTRM) phase function provided the best fit to the experimental results. We further validated the TTRM approximation for the case of single scattering by comparing the TTRM function to calculations by Mie theory. We found that the TTRM is a good approximation for the Rayleigh and Mie scattering regimes, up to size parameters of 2 to 3 , and can even be extended in an average sense to the geometric optics regime. In case of multiple scattering, the oscillations in the phase function will diminish and the TTRM function is expected to be valid in an even more extended range of size parameters. In particular we have shown that it gives an excellent description to the case of phase functions that are strongly peaked in both the forward and backward directions. Our results hence establish a useful approximation to the scattering phase function of metallic and dielectric nanoparticles and composites, which can be used for model calculations in a number of applications such as pigmented coatings, marine scatterers, biological materials, and particulate air contaminants.

\section{Experimental}

\section{Sample synthesis and characterization}

The three types of nanoparticle samples were prepared using a bottom-up wet chemistry method, which allows for good control of the shape and size of nanostructures. Au nanospheres (NSs) were obtained by a seed-mediated growth together with mild oxidation, starting from small Au NSs, growing to nanopolyhedra, and reshaping to $\sim 200 \mathrm{~nm}$ diameter Au NSs by etching the edges. ${ }^{36}$ In a typical synthesis of $\mathrm{Fe}_{3} \mathrm{O}_{4} \mathrm{NSs}, \mathrm{FeCl}_{3} \cdot 6 \mathrm{H}_{2} \mathrm{O}(0.1 \mathrm{~g})$, polyvinylpyrrolidone (PVP, MW: $40000,4 \mathrm{~g})$, sodium acetate trihydrate $\left(\mathrm{NaAc} \cdot 3 \mathrm{H}_{2} \mathrm{O}, 0.3 \mathrm{~g}\right)$ and $1 \mathrm{~mL}$ of polyethylene glycol (PEG, MW: 300) were dissolved in $20 \mathrm{~mL}$ of ethylene glycol under stirring and ultrasonic treatment. The homogeneous yellow mixture was then transferred to a $50 \mathrm{~mL}$ Teflon-lined stainless-steel autoclave which was sealed and heated to $200{ }^{\circ} \mathrm{C}$ for $4 \mathrm{~h}$. The $\mathrm{Fe}_{3} \mathrm{O}_{4}$ NSs were formed and collected by a magnet, washed with $50 \mathrm{~mL}$ distilled water, and collected by centrifugation at $1700 \mathrm{rpm}$ for $20 \mathrm{~min}$. The washing and centrifugation processes were repeated 5 times. The $\mathrm{Fe}_{3} \mathrm{O}_{4}$ NSs were eventually obtained after drying overnight at $80{ }^{\circ} \mathrm{C}$. $\mathrm{TiO}_{2}$ NSs were synthesized using a similar procedure previously described by $\mathrm{Han}^{37}$ with minor modifications. Briefly, $200 \mu \mathrm{L}$ of $\mathrm{CaCl}_{2}$ solution $(0.05 \mathrm{M})$ was added to $50 \mathrm{~mL}$ methanol into a $100 \mathrm{~mL}$ one-necked flask and stirred for $10 \mathrm{~min}$. Then $850 \mu \mathrm{L}$ of titanium(Iv) isopropoxide was added dropwise. The resulting solution was magnetically stirred for $24 \mathrm{~h}$ at room temperature. The synthesized $\mathrm{TiO}_{2} \mathrm{NSs}$ were subsequently washed with $50 \mathrm{~mL}$ distilled water and collected by centrifugation at $1700 \mathrm{rpm}$ for $20 \mathrm{~min}$. The washing and centrifugation processes were repeated 5 times. The $\mathrm{TiO}_{2}$ NSs were obtained by calcination at $450^{\circ} \mathrm{C}$ for $2 \mathrm{~h}$ with a ramp rate of $5{ }^{\circ} \mathrm{C} \mathrm{min}^{-1}$.

The $\mathrm{Au}, \mathrm{Fe}_{3} \mathrm{O}_{4}$ and $\mathrm{TiO}_{2}$ NSs were added to water at a concentration of $19.3 \mathrm{mg} \mathrm{mL}{ }^{-1}, 5 \mathrm{mg} \mathrm{mL} \mathrm{m}^{-1}$ and $4 \mathrm{mg} \mathrm{mL}^{-1}$, corresponding to $0.1 \%$ in volume fraction, equivalent to a particle concentration of $2.39 \times 10^{11} \mathrm{~mL}^{-1}$. Then PVP was added into the NS/water solution at a mass ratio of 0.8 (PVP/water). PVP has high viscosity, good binding capability with water, it dissolves at room temperature and is highly stable and nontoxic. The NS/water/PVP solution was vigorously shaken in a vortex mixer until the PVP powders dissolved completely. The viscous solution was deposited on a glass slide, subsequently another glass side with a spacer $\sim 80 \mu \mathrm{m}$ in thickness was added on top. The particle-PVP composite sample was sealed with glue and measurements took place after two weeks when the samples had stabilized. Significant particle aggregation can be found in the nanoparticle composites when studied under the microscope (Fig. S11†).

Scanning electron microscope images (Fig. S1 $\dagger$ ) were acquired by a Zeiss (LEO) 1530 FEG microscope at an accelera- 
tion voltage of $5 \mathrm{keV}$. Particle diameters and size distributions were determined by Adobe Illustrator software.

\section{Optical characterization}

The 'in-plane' angular and spectral resolved spectrometer consisted of a tungsten-halogen lamp, a monochromator, two gratings, an off-axis parabolic aluminum mirror with an offaxis distance of $80 \mathrm{~mm}$ and a focal length of $410 \mathrm{~mm}$. The detector was a silicon diode with a spectral response region from $300 \mathrm{~nm}$ to $1100 \mathrm{~nm}$ and a port dimension of $6.4 \times$ $6.4 \mathrm{~mm}^{2}$. The sample was centrally mounted and illuminated, and the silicon detector could be positioned at various angles from $0^{\circ}$ to $180^{\circ}$. To increase the dynamic range of the measurement, we applied different neutral density filters (Thorlabs) for measurements at different wavelengths. These filters are designed for $633 \mathrm{~nm}$. A 50\% filter was used for $350 \mathrm{~nm}$ (transmittance of 0.16258), a 1\% filter was used for $400 \mathrm{~nm}$ (transmittance: 0.00266), $500 \mathrm{~nm}$ (transmittance: 0.00653 ), $600 \mathrm{~nm}$ (transmittance: 0.00747 ), a $0.1 \%$ filter was used for $700 \mathrm{~nm}$ (transmittance: 0.00512), $800 \mathrm{~nm}$ (transmittance: 0.01159), $900 \mathrm{~nm}$ (transmittance: 0.01138), $1000 \mathrm{~nm}$ (transmittance: 0.01012 ). The incident light spot was much smaller than the port of the Si detector. The incident light intensity $P_{\mathrm{i}}$ was calculated using the measured intensity with the filter $I_{\text {filter }}$, divided by the filter transmittance at the specific wavelength. The light spot spanned an angle within $\pm 1^{\circ}$. The transmittance and reflectance were measured as a function of angle at a fixed wavelength. An angle of $10^{\circ}$ of the sample normal to the incident light was used for reflectance measurements, to be able to measure the specular and near-specular reflected intensity, subsequently the data were shifted by Harvey's method. ${ }^{38}$ The measured radiant intensity was represented in the form of angle resolved scattering (ARS), by normalizing with the incident light intensity $P_{\mathrm{i}}$ and the solid angle $\Omega$ of the detector: ${ }^{3}$

$$
\operatorname{ARS}(\theta)=\frac{S(\theta)}{P_{\mathrm{i}} \times \Omega}=\frac{S(\theta)}{\frac{S\left(0^{\circ}\right)}{T_{\text {filter }}} \times \frac{A}{R^{2}}}
$$

where $S(\theta)$ is the measured radiant intensity of the sample. In the small-angle region, i.e., smaller than $2^{\circ}$, the measurements were conducted together with a filter and thereby the intensity was divided by the filter transmittance. $S\left(0^{\circ}\right)$ is the measured intensity of the incident light with filter and without sample. $T_{\text {filter }}$ is the transmittance of the filter at the desired wavelength, $A$ is the surface area of the detector port, $R$ is the distance from the sample to the detector, i.e. $20 \mathrm{~cm}$. The solid angle $\Omega$ was equal to $6.4^{2} / 200^{2}=0.001024 \mathrm{sr}^{-1}$.

The 'out-of-plane' spatial scattering intensity distribution was measured using a home-built goniometer. ${ }^{39}$ The light source was a red He-Ne laser (wavelength $\lambda=633 \mathrm{~nm}$ ). The position-controlled detector was mounted on a movable arm, thereby allowing the detector to move in a hemisphere. The radius of the semicircular arc was $45 \mathrm{~cm}$. The sample was fixed at the center of the hemisphere traced by the movable arm, and was free to rotate around a horizontal axis, to vary the angle of incidence. The sample holder, the detector, and the arc were controlled by stepping motors. We illuminated a relatively small spot on the sample and measured the amount of light scattered into a known solid angle (under-illumination method). ${ }^{3}$ The detector was a silicon diode with a spectral response region from $300 \mathrm{~nm}$ to $1100 \mathrm{~nm}$ with port dimension $2.5 \times 2.4 \mathrm{~mm}^{2}$. The incident light intensity was measured using a neutral density filter with a transmittance of 0.0004821 at $633 \mathrm{~nm}$. In transmittance mode, the incident light was parallel to the normal of the sample surface. In the reflectance mode, the incident light was incident at $10^{\circ}$ to the sample normal, the same as in the in-plane scattering measurement. The position of the sample to the incident light was fixed, and we measured a half hemisphere both in transmittance and reflectance regions and mirrored their intensity distributions to the other half hemisphere. The data analysis was similar to the in-plane spectrometer; the intensity was divided by the incident light intensity $P_{\mathrm{i}}$ and the solid angle $\Omega$ of the detector port. The arm moved from $-90^{\circ}$ to $90^{\circ}$ while the detector moved in the range from $-68^{\circ}$ to $68^{\circ}$ on the arm due to the geometry of the instrument. The measured data are presented as a function of real three dimensional position, $(x, y, z)$. Then we convert the real position $(x, y, z)$ into the polar $(\theta)$ and azimuth angles $(\varphi)$ by,

$$
\begin{gathered}
\theta=\arctan \left(\frac{\sqrt{x^{2}+y^{2}}}{z}\right) \\
\varphi=\arctan \left(\frac{y}{x}\right)
\end{gathered}
$$

\section{Mie theory modeling}

The electrodynamic response of particles of spherical shape can be analytically and effectively solved with Mie theory. ${ }^{21}$ The scattering phase function for the three types of nanospheres was computed using the program MiePlot version 4.6. ${ }^{40}$ The calculations by Mie theory took into consideration the particle size distributions measured from SEM images (Fig. $\mathrm{S} 1 \dagger$ ). The refractive index database of $\mathrm{Au},{ }^{41} \mathrm{Fe}_{3} \mathrm{O}_{4}{ }^{42}$ and $\mathrm{TiO}_{2}{ }^{43}$ were used as input for the optical properties of the nanospheres while the optical constants of the surrounding medium were taken from the calculated $n, k$ obtained from $T_{\text {reg }}$ and $R_{\text {spe }}$ of a glass/(water + PVP)/glass sample, using the method of McPhedran et al. ${ }^{44}$

Mie theory was used to calculate the far-field scattering pattern around the particle. ${ }^{15}$ In Mie theory, ${ }^{15}$ the scattering phase function $F(\theta, \varphi)$ defines the intensity of the scattered light in an arbitrary direction.

$$
F(\theta, \varphi)=i_{2}(\theta) \cos ^{2}(\varphi)+i_{1}(\theta) \sin ^{2}(\varphi)
$$

Where $i_{1}=\left|S_{1}(\theta)\right|^{2}$ and $i_{2}=\left|S_{2}(\theta)\right|^{2}$ and the amplitude functions $S_{1}(\theta)$ and $S_{2}(\theta)$ are complicated functions of the size parameter $x$ as well as the ratio of the refractive indices of the particle and the surrounding medium. Hence the scattering behaviour is influenced only by the diameter of the nanoparticle, the incident wavelength, and the refractive index of the particle and the medium. 


\section{Conflicts of interest}

There are no conflicting interests to declare.

\section{Acknowledgements}

This work was financially supported by the Swedish Research Council grant 2016-03713. We are grateful to Nanoseedz Reagent Co., Ltd (Hong Kong, China) for supplying the Au nanospheres.

\section{References}

1 J. C. Stover, Optical scattering: measurement and analysis, SPIE Optical Engineering Press, Bellingham, WA, USA, 1995.

2 A. K. Fung and H. J. Eom, IEEE Trans. Geosci. Remote Sens., 1982, GE-20, 528-536.

3 T. A. Germer, J. C. Zwinkels and B. K. Tsai, Spectrophotometry: Accurate measurement of optical properties of materials, Elsevier Science, Amsterdam, The Netherlands, 2014.

4 Lord Rayleigh, Proc. R. Soc. London, Ser. A, 1907, 79, 399-416.

5 S. O. Rice, Commun. Pur. Appl. Math., 1951, 4, 351-378.

6 S. Schröder, T. Herffurth, H. Blaschke and A. Duparré, Appl. Opt., 2011, 50, C164-C171.

7 C. Liu, R. L. Panetta and P. Yang, J. Quant. Spectrosc. Radiat. Transfer, 2013, 129, 169-185.

8 C. Li, G. W. Kattawar and P. Yang, J. Quant. Spectrosc. Radiat. Transfer, 2004, 89, 123-131.

9 P. Kubelka and F. Munk, Z. Tech. Phys., 1931, 12, 593-601.

10 V. I. Haltrin, Appl. Opt., 2002, 41, 1022-1028.

11 C. Gabriel, M.-A. Khalighi, S. Bourennane, P. Léon and V. Rigaud, J. Opt. Commun. Netw., 2013, 5, 1-12.

12 A. J. Baran, V. N. Shcherbakov, B. A. Baker, J. F. Gayet and R. P. Lawson, Q. J. R. Meteorol. Soc., 2005, 131, 2609-2616.

13 P. Thueler, I. Charvet, F. Bevilacqua, M. St. Ghislain, G. Ory, P. Marquet, P. Meda, B. Vermeulen and C. Depeursinge, J. Biomed. Opt., 2003, 8, 495-503.

14 S. L. Jacques, C. A. Alter and S. A. Prahl, Lasers Life Sci., 1987, 4, 309-334.

15 H. C. van de Hulst, Light Scattering by Small Particles, Wiley, New York, 1957.

16 D. Toublanc, Appl. Opt., 1996, 35, 3270-3274.

17 U. Kreibig and M. Vollmer, Optical properties of metal clusters, Springer-Verlag, Berlin, 1995.

18 H. Chen, L. Shao, Q. Li and J. Wang, Chem. Soc. Rev., 2013, 42, 2679-2724.
19 B. Tian, J. Ma, Z. Qiu, T. Zardán Gómez de la Torre, M. Donolato, M. F. Hansen, P. Svedlindh and M. Strömberg, ACS Nano, 2017, 11, 1798-1806.

20 F. Huang, D. Chen, X. L. Zhang, R. A. Caruso and Y.-B. Cheng, Adv. Funct. Mater., 2010, 20, 1301-1305.

21 C. Bohren and D. R. Huffman, Absorption and Scattering of Light by Small Particles, John Wiley \& Sons, New York, 1998.

22 B. C. Wilson and G. Adam, Med. Phys., 1983, 10, 824-830.

23 Z. Liu, K. Wang, X. Luo and S. Liu, Opt. Express, 2010, 18, 9398-9412.

24 W. Sun, Q. Fu and Z. Chen, Appl. Opt., 1999, 38, 3141-3151.

25 L. C. Henyey and J. L. Greenstein, Astrophys. J., 1941, 93, 70-83.

26 P. Laven, Appl. Opt., 2005, 44, 5675-5683.

27 G. W. Kattawar, J. Quant. Spectrosc. Radiat. Transfer, 1975, 15, 839-849.

28 W. M. Cornette and J. G. Shanks, Appl. Opt., 1992, 31, 3152-3160.

29 L. O. Reynolds and N. J. McCormick, J. Opt. Soc. Am., 1980, 70, 1206-1212.

30 G. R. Fournier and J. L. Forand, Proc. SPIE, 1994, 2258, 194-201.

31 I. Gkioulekas, B. Xiao, S. Zhao, E. H. Adelson, T. Zickler and K. Bala, ACM Trans. Graph., 2013, 32, 1-19.

32 H. C. van de Hulst, Multiple light scattering: tables, formulas, and applications, Academic, New York, 1980.

33 J. Piskozub and D. McKee, Opt. Express, 2011, 19, 47864794.

34 E. Akkermans, P. E. Wolf and R. Maynard, Phys. Rev. Lett., 1986, 56, 1471-1474.

35 L. Tsang and A. Ishimaru, J. Opt. Soc. Am. A, 1984, 1, 836839.

36 Q. Ruan, L. Shao, Y. Shu, J. Wang and H. Wu, Adv. Opt. Mater., 2014, 2, 65-73.

37 C. Han, R. Luque and D. D. Dionysiou, Chem. Commun., 2012, 48, 1860-1862.

38 J. E. Harvey, Proc. SPIE, 1990, 1165, 87-99.

39 M. Rönnelid, M. Adsten, T. Lindström, P. Nostell and E. Wäckelgård, Appl. Opt., 2001, 40, 2148-2158.

40 P. Laven, 2018, http://www.philiplaven.com/mieplot.htm.

41 S. Babar and J. H. Weaver, Appl. Opt., 2015, 54, 477-481.

42 A. Schlegel, S. F. Alvarado and P. Wachter, J. Phys. C: Solid State Phys., 1979, 12, 1157-1164.

43 L. Miao, P. Jin, K. Kaneko, A. Terai, N. Nabatova-Gabain and S. Tanemura, Appl. Surf. Sci., 2003, 212-213, 255-263.

44 R. C. McPhedran, L. C. Botten, D. R. McKenzie and R. P. Netterfield, Appl. Opt., 1984, 23, 1197-1205. 